\title{
A multistream model for quantum plasmas
}

\author{
F. Haas $^{1}$, G. Manfredi ${ }^{2}$ \\ Laboratoire de Physique des Milieux Ionisés, Université Henri Poincaré, \\ BP 239, 54506 Vandoeuvre-les-Nancy, France \\ M. Feix \\ Subatech, Ecole des Mines de Nantes, BP 20722, 44397 Nantes Cedex 3, France
}

\begin{abstract}
The dynamics of a quantum plasma can be described self-consistently by the nonlinear Schrödinger-Poisson system. Here, we consider a multistream model representing a statistical mixture of $N$ pure states, each described by a wavefunction. The one-stream and two-stream cases are investigated. We derive the dispersion relation for the two-stream instability and show that a new, purely quantum, branch appears. Numerical simulations of the complete Schrödinger-Poisson system confirm the linear analysis, and provide further results in the strongly nonlinear regime. The stationary states of the Schrödinger-Poisson system are also investigated. These can be viewed as the quantum mechanical counterpart of the classical Bernstein-Greene-Kruskal modes, and are described by a set of coupled nonlinear differential equations for the electrostatic potential and the stream amplitudes.

${ }^{1}$ Fernando.Haas@lpmi.uhp-nancy.fr

${ }^{2}$ Giovanni.Manfredi@lpmi.uhp-nancy.fr
\end{abstract}




\section{Introduction}

The great degree of miniaturization of today's electronic components is such that the de Broglie wavelength of the charge carriers is frequently comparable to the dimensions of the system. Hence, quantum mechanical effects (e.g. tunneling) are expected to play a central role in the behavior of electronic components to be constructed in the next years. In order to describe these quantum effects, it is unlikely that classical transport models will be sufficient. Quantum transport equations, such as the Schrödinger-Poisson or the Wigner-Poisson systems [1]-[0], will therefore be a necessary tool in order to understand the basic properties of these physical systems.

In the present paper, we consider a one-dimensional quantum plasma, where the electrons are described by a statistical mixture of $N$ pure states, with each wavefunction $\psi_{i}$ obeying the Schrödinger-Poisson system

$$
\begin{aligned}
i \hbar \frac{\partial \psi_{i}}{\partial t} & =-\frac{\hbar^{2}}{2 M} \frac{\partial^{2} \psi_{i}}{\partial x^{2}}-e \phi \psi_{i}, \quad i=1, \ldots, N \\
\frac{\partial^{2} \phi}{\partial x^{2}} & =\frac{e}{\varepsilon_{0}}\left(\sum_{i=1}^{N}\left|\psi_{i}\right|^{2}-n_{0}\right),
\end{aligned}
$$

where $\phi(x, t)$ is the electrostatic potential. Electrons have mass $M$ and charge $-e$, and are globally neutralized by a fixed ion background with density $n_{0}$. Finally, we assume periodic boundary conditions, with spatial period equal to $L$.

The system of Eqs. (11)-(2) takes into account diffraction, which is the most prominent quantum effect, but neglects dissipation, spin and relativistic corrections. These effects may be important in more realistic models for small semiconductor devices. Nevertheless, it is useful to consider simplified models that capture the main features of quantum plasmas. Indeed, Eqs. (11)-(2) are sufficiently rich to display a wide variety of behaviors, as it will be seen in the rest of this work. At the same time, this model is still amenable to analytic and numerical treatment.

A physically equivalent approach would consist in considering a Wigner function describing the same mixture. The Wigner function approach is a reformulation of quantum mechanics in the classical phase space language [8]- [10]. The price to pay for this otherwise appealing formalism, is that Wigner functions can take negative values, and cannot therefore be regarded as true probability distributions. However, both for the analytical and the numerical treatment of the problems of interest in this paper, the Schrödinger-Poisson formalism is more appropriate. This is particularly true for the numerical simulations, since the Wigner formalism is cast into a two-dimensional phase space, whilst the Schrödinger-Poisson model only requires the discretization of the one-dimensional configuration space. Of 
course, if the number $N$ of streams is large, the numerical cost for the description of the system of Eqs. (11)-(2) is also considerable. Nevertheless, interesting physical phenomena (such as instabilities) can take place even with a few streams $(N=1$ or 2 ), as it will appear in the rest of this work. More subtle effects (Landau damping, for instance) would probably require a larger, although hopefully not prohibitive, number of streams $(N \simeq 20-30)$.

For the analytical study, the hydrodynamic formulation of the Schrödinger-Poisson system is particularly convenient, since it makes direct use of macroscopic plasma quantities, such as density and average velocity. Moreover, it enables one to perform straightforward perturbation calculations in the same fashion as in the classical case. Let us introduce the amplitude $A_{i}$ and the phase $S_{i}$ associated to the pure state $\psi_{i}$ according to

$$
\psi_{i}=A_{i} \exp \left(i S_{i} / \hbar\right)
$$

Both $A_{i}$ and $S_{i}$ are defined as real quantities. The density $n_{i}$ and the velocity $u_{i}$ of the $i$-th stream of the plasma are given by

$$
n_{i}=A_{i}^{2}, \quad u_{i}=\frac{1}{M} \frac{\partial S_{i}}{\partial x}
$$

Introducing Eqs. (3)-(国) into Eqs. (11)-(2) and separating the real and imaginary parts of the equations, we find

$$
\begin{aligned}
\frac{\partial n_{i}}{\partial t}+\frac{\partial}{\partial x}\left(n_{i} u_{i}\right) & =0 \\
\frac{\partial u_{i}}{\partial t}+u_{i} \frac{\partial u_{i}}{\partial x} & =\frac{e}{M} \frac{\partial \phi}{\partial x}+\frac{\hbar^{2}}{2 M^{2}} \frac{\partial}{\partial x}\left(\frac{\partial^{2}\left(\sqrt{n_{i}}\right) / \partial x^{2}}{\sqrt{n_{i}}}\right), \\
\frac{\partial^{2} \phi}{\partial x^{2}} & =\frac{e}{\varepsilon_{0}}\left(\sum_{i=1}^{N} n_{i}-n_{0}\right) .
\end{aligned}
$$

Quantum effects are contained in the pressure-like, $\hbar$-dependent term in Eq. (6). If we set $\hbar=0$, we simply obtain the classical multistream model introduced by Dawson [11]. Therefore, we shall refer to Eqs. (5)-(7) as the quantum multistream model.

Equations (5)-(7) constitute the mathematical model used in the rest of this work. We focus our attention on the one-stream (Sec. 2) and two-stream (Sec. 3) cases, the latter being related to the well-known two-stream instability. The relevant dispersion relations are derived, and the unstable branches are identified. We also investigate the properties of stationary solutions of the Schrödinger-Poisson system, which can be viewed as the quantum counterpart of the classical BernsteinGreene-Kruskal (BGK) modes [12]. The analytical calculations are checked against time-dependent numerical simulations, shown in Sec. 4. Our conclusions are presented in Sec. 5. 


\section{One-stream plasma}

In order to fix the basic ideas, we first consider the one-stream case and take $N=1$, i.e. a single pure quantum state. For brevity, we write $n_{1} \equiv n, u_{1} \equiv u$. We obtain

$$
\begin{aligned}
\frac{\partial n}{\partial t}+\frac{\partial}{\partial x}(n u) & =0 \\
\frac{\partial u}{\partial t}+u \frac{\partial u}{\partial x} & =\frac{e}{M} \frac{\partial \phi}{\partial x}+\frac{\hbar^{2}}{2 M^{2}} \frac{\partial}{\partial x}\left(\frac{\partial^{2}(\sqrt{n}) / \partial x^{2}}{\sqrt{n}}\right), \\
\frac{\partial^{2} \phi}{\partial x^{2}} & =\frac{e}{\varepsilon_{0}}\left(n-n_{0}\right) .
\end{aligned}
$$

A homogeneous zeroth-order solution for Eqs. (8)-(10) is provided by

$$
n=n_{0}, \quad u=u_{0}, \quad \phi=0,
$$

where $u_{0}$ is a constant representing the equilibrium velocity of the stream. The linear stability of this solution is obtained by Fourier analyzing Eqs. (8)-(10),

$$
\begin{aligned}
n & =n_{0}+n^{\prime} \exp (i(k x-\omega t)), \\
u & =u_{0}+u^{\prime} \exp (i(k x-\omega t)), \\
\phi & =\phi^{\prime} \exp (i(k x-\omega t))
\end{aligned}
$$

Retaining only terms up to first order in $n^{\prime}, u^{\prime}$ and $\phi^{\prime}$ leads to the dispersion relation

$$
\left(\omega-k u_{0}\right)^{2}=\omega_{p}^{2}+\hbar^{2} k^{4} / 4 M^{2},
$$

where $\omega_{p}=\left(n_{0} e^{2} / M \varepsilon_{0}\right)^{1 / 2}$ is the plasma frequency. For $u_{0}=0$, the dispersion relation given in 13 is recovered, the term $k u_{0}$ merely representing a Doppler shift. As the frequency $\omega$ is always real, there can be neither instability nor damping of the wave.

The classical analog of this system is the "cold plasma" model, which is known to sustain nonlinear oscillations when the amplitude of the initial perturbation is smaller than a certain value. Beyond that value, the solution becomes singular in a finite time, which is a sign that the model is no longer valid. This phenomenon corresponds to the breaking of the plasma wave, due to particle overtaking in the phase space. Due to the pressure-like term in Eq. (9), the quantum solution nevers becomes singular, as it was shown by computer simulations [14].

Let us now turn our attention to the stationary regimes of the system. If all quantities only depend on position, then Eqs. (8)-(9) are reduced to

$$
\begin{aligned}
\frac{d}{d x}(n u) & =0 \\
u \frac{d u}{d x} & =\frac{e}{M} \frac{d \phi}{d x}+\frac{\hbar^{2}}{2 M^{2}} \frac{d}{d x}\left(\frac{d^{2}(\sqrt{n}) / d x^{2}}{\sqrt{n}}\right) .
\end{aligned}
$$


Equations (16)-(17) possess the first integrals

$$
\begin{aligned}
J & =n u, \\
E & =\frac{M u^{2}}{2}-e \phi-\frac{\hbar^{2}}{2 M}\left(\frac{d^{2}(\sqrt{n}) / d x^{2}}{\sqrt{n}}\right),
\end{aligned}
$$

corresponding to charge and energy conservation. The constant $E$ can be eliminated by the global shift $\phi \rightarrow \phi+E / e$, and therefore we assume $E=0$ in the rest of this section. Eliminating $u$, introducing $A=\sqrt{n}$ and using Poisson's equation, we obtain

$$
\begin{aligned}
\hbar^{2} \frac{d^{2} A}{d x^{2}} & =M\left(\frac{M J^{2}}{A^{3}}-2 e A \phi\right) \\
\frac{d^{2} \phi}{d x^{2}} & =\frac{e}{\varepsilon_{0}}\left(A^{2}-n_{0}\right) .
\end{aligned}
$$

It can be easily verified that the $J=0$ case cannot sustain small-amplitude, periodic solutions. Hence, we assume $J=n_{0} u_{0}$ with $u_{0} \neq 0$ and introduce the following rescaling

$$
\begin{aligned}
x^{*} & =\frac{\omega_{p} x}{u_{0}}, \quad A^{*}=\frac{A}{\sqrt{n_{0}}}, \\
\phi^{*} & =\frac{e \phi}{M u_{0}^{2}}, \quad H=\frac{\hbar \omega_{p}}{M u_{0}^{2}} .
\end{aligned}
$$

We obtain, in the transformed variables (omitting the stars for simplicity of notation),

$$
\begin{aligned}
H^{2} \frac{d^{2} A}{d x^{2}} & =-2 \phi A+\frac{1}{A^{3}}, \\
\frac{d^{2} \phi}{d x^{2}} & =A^{2}-1,
\end{aligned}
$$

a system that only depends on the rescaled parameter $H$, which is a measure of the importance of quantum effects.

Notice that the classical limit is singular, in the sense that Eq. (23) degenerates into an algebraic equation when $H=0$. The equation for the electrostatic potential then becomes

$$
\frac{d^{2} \phi}{d x^{2}}=\frac{1}{\sqrt{2 \phi}}-1 .
$$

Equation (25) has a Hamiltonian character, and corresponds to the equation of motion of a "particle" moving in a potential $V(\phi)=\phi-\sqrt{2 \phi}$. Using this analogy, one can see that Eq. (25) has periodic solutions around the equilibrium $\phi=1 / 2$, 
provided that the initial condition satisfies $0<\phi(x=0)<2$. The fact that no solution exists for sufficiently large values of the potential is easily understood. A large potential fluctuation induces a velocity fluctuation, which can drive $u(x)$ far from its nominal value $u_{0}$. If the potential is sufficiently strong, $u(x)$ can even vanish, but in that case the relation $n u=J=$ constant implies an infinite density. This is the well-known effect of particle overtaking that occurs in the cold plasma model.

Going back to the quantum mechanical case, we have shown that Eqs. (23)(24) describe the inhomogeneous QBGK (quantum-BGK) equilibria of the onecomponent quantum plasma. We are faced with the mathematical problem of understanding the qualitative properties of the solutions of a coupled, nonlinear system of two second order differential equations depending on a parameter. Only few analytical results can be obtained, shown in the rest of this section.

Equations (23)-(24) can be put into Hamiltonian form by using the variables

$$
\bar{A}=i A, \quad \bar{\phi}=\phi / H .
$$

Notice that the rescaled amplitude $\bar{A}$ is a purely imaginary quantity. We have

$$
d^{2} \bar{A} / d x^{2}=-\partial U / \partial \bar{A}, \quad d^{2} \bar{\phi} / d x^{2}=-\partial U / \partial \bar{\phi},
$$

where $U \equiv U(\bar{A}, \bar{\phi})$ is the pseudo-potential

$$
U(\bar{A}, \bar{\phi})=\frac{1}{H}\left(1+\bar{A}^{2}\right) \bar{\phi}+\frac{1}{2 H^{2} \bar{A}^{2}} .
$$

Since the equations of motion are autonomous with respect to the independent variable $x$, the Hamiltonian formulation immediately gives the first integral (subscripts denote differentiation)

$$
I=\frac{1}{2}\left(\bar{A}_{x}^{2}+\bar{\phi}_{x}^{2}\right)+U(\bar{A}, \bar{\phi})
$$

which is the Hamiltonian function in transformed coordinates. Transforming back to the original variables, one obtains the first integral for Eqs. (23)-(24)

$$
I=\frac{1}{2}\left(-A_{x}^{2}+H^{-2} \phi_{x}^{2}\right)+\frac{1}{H^{2}}\left(1-A^{2}\right) \phi-\frac{1}{2 H^{2} A^{2}} .
$$

According to Liouville-Arnold theorem [15], an autonomous two-degree of freedom Hamiltonian system is completely integrable if it possesses two first integrals in involution and with compact level surfaces. Even if $I$ has not compact level surfaces, a second constant of motion would be a strong indication of integrability of the QBGK spatial dynamics. We have tried to find a second constant of motion 
for Eqs. (23)-(24) by a variety of methods (geometrical Noether and Lie symmetries, for instance), but without success. However, numerical integrations of Eqs. (23) -(24) for a wide range of values of $H$, and different initial conditions, strongly suggest that, when bounded solutions exist, they are always regular. An additional first integral must therefore exist, although its actual expression may be difficult to guess.

It is interesting to perform a linear stability analysis in order to see in what conditions the system supports small amplitude spatially periodic solutions. Writing

$$
A=1+A^{\prime} \exp (i k x), \quad \phi=1 / 2+\phi^{\prime} \exp (i k x)
$$

and retaining in Eqs. (23)-(24) only terms up to first order in the primed variables, we obtain the relation

$$
H^{2} k^{4}-4 k^{2}+4=0 .
$$

Again, we point out the singular character of the classical limit: for $H=0$, Eq. (32) degenerates into a quadratic equation, with solutions $k= \pm 1$. The wavenumbers being always real, this corresponds to spatially periodic solutions. When $H \neq 0$, we obtain

$$
k^{2}=\frac{2 \pm 2 \sqrt{1-H^{2}}}{H^{2}} .
$$

For $H<1$ (semiclassical regime), both wavenumbers are real, and therefore the system can sustain spatially periodic oscillations. For $H>1$ (strong quantum effects), the solutions are spatially unstable, and grow exponentially. For $H=1$, the spectrum is degenerate, with associated secular terms. The corresponding solution is also spatially unstable, growing linearly with $x$. We conclude that smallamplitude stationary solutions of the one-stream Schrödinger-Poisson system can only exist in the semiclassical regime, $H<1$.

\section{Two-stream plasma}

\subsection{Two-stream instability}

We now turn to the more interesting case of two streams, which classically can give rise to instability. For this, we consider Eqs. (5)-(7) with $N=2$. We first linearize around the equilibrium solution $n_{1}=n_{2}=n_{0} / 2, u_{1}=-u_{2}=u_{0}, \phi=0$, where $u_{0} \neq 0$ is a nonzero reference velocity, and then Fourier transform both in space and time variables. In terms of the dimensionless variables

$$
\Omega=\omega / \omega_{p}, \quad K=u_{0} k / \omega_{p}, \quad H=\hbar \omega_{p} / M u_{0}^{2},
$$


the dispersion relation becomes

$$
\Omega^{4}-\left(1+2 K^{2}+\frac{H^{2} K^{4}}{2}\right) \Omega^{2}-K^{2}\left(1-\frac{H^{2} K^{2}}{4}\right)\left(1-K^{2}+\frac{H^{2} K^{4}}{4}\right)=0 .
$$

Consequently, one obtains

$$
\Omega^{2}=\frac{1}{2}+K^{2}+\frac{H^{2} K^{4}}{4} \pm \frac{1}{2}\left(1+8 K^{2}+4 H^{2} K^{6}\right)^{1 / 2}
$$

The solution for $\Omega^{2}$ has two branches, one of which is always positive and gives stable oscillations. The other solution is negative $\left(\Omega^{2}<0\right)$ provided that

$$
\left(H^{2} K^{2}-4\right)\left(H^{2} K^{4}-4 K^{2}+4\right)<0
$$

Notice that instability, when it occurs, always arises through the marginal mode $(\Omega=0)$. In the classical case, Eq. (37) yields $K^{2}<1$, which is the classical criterion for the occurrence of the two-stream instability. In the quantum case, Eq. (37) bifurcates for $H=1$. If $H>1$, the second factor is always positive, and the plasma is unstable if $H K<2$. If $H<1$, there is instability if either

$$
0<H^{2} K^{2}<2-2 \sqrt{1-H^{2}}
$$

or

$$
2+2 \sqrt{1-H^{2}}<H^{2} K^{2}<4 .
$$

This yields the stability diagram plotted on Fig. 1. The lower instability zone is semiclassical, as it represents an extension of the classical instability criterion. The upper instability zone, on the other hand, has no classical analog. The two zones merge for $H=1$. We call $K_{A}, K_{B}$ and $K_{C}$ the wavenumbers at which the growth rate vanishes. These are defined by

$$
\begin{aligned}
H^{2} K_{A, B}^{2} & =2 \pm 2 \sqrt{1-H^{2}} \\
H^{2} K_{C}^{2} & =4 .
\end{aligned}
$$

It is easy to verify the following property

$$
K_{A}^{2}+K_{B}^{2}=K_{C}^{2}
$$

We shall see that these wavelengths are related to the stationary solutions of the Schrödinger-Poisson system (quantum-BGK, or QBGK, modes). ¿From the previous analysis, it appears that quantum mechanics has a destabilizing effect in the semiclassical regime, where more modes are unstable compared to the classical case. On the other hand, when $H>2$, fewer modes turn out to be unstable than 
in the classical regime. This is the result obtained by Suh, Feix and Bertrand [16], who found that quantum effects are stabilizing for large enough $H$.

It would be interesting to know whether the quantum instability is stronger or weaker than the classical one. For this, one should search for the maximum (over all wavenumbers) growth rate for a fixed value of $H$, and compare it to the maximum classical growth rate. Although the algebra is rather involved, direct inspection of the function $\gamma(K)$ for different values of $H$ indicates that the maximum classical growth rate is always larger than the maximum quantum growth rate (Fig. 2) [on this figure, the intersections with the $K$ axis correspond to wavenumbers $K_{A}$, $K_{B}$ and $K_{C}$ as defined in Eqs. (40)-(41), for which the growth rate vanishes]. Of course, for some wavenumbers, the quantum growth rate may be larger than the classical one, as can be seen from Fig. 2. Note that the secondary maximum (between wavenumbers $K_{B}$ and $K_{C}$ ) is considerably smaller than the one between $K=0$ and $K_{A}$, and goes to zero in the classical limit $H \rightarrow 0$.

In the previous discussion, we have ignored the fact that, due to the periodic boundary conditions, the momentum variable $p=\hbar k$ is discrete, and can only be a multiple of $\hbar k_{0}$, where $k_{0}=2 \pi / L$ is the fundamental wavenumber. For the previous stability calculations, we have assumed an initial equilibrium solution for which the density $n_{0}$ is spatially uniform and the velocity is equal to $\pm u_{0}$. This corresponds to wavefunctions of the type

$$
\psi_{ \pm}(x, 0)=\sqrt{n_{0} / 2} \exp \left( \pm i M u_{0} x / \hbar\right) .
$$

In order to satisfy the periodicity, one must have

$$
M u_{0}=n \hbar k_{0}, \quad n=1,2,3 \ldots
$$

Using the dimensionless variables introduced earlier, this condition becomes

$$
H K_{0}=1 / n
$$

which implies $H K_{0} \leq 1$. This result sets an upper bound on the fundamental wavenumber $K_{0}$. On the instability diagram of Fig. 1, this means that the upper instability region cannot be accessed for $K_{0}$, although of course it can for some of its harmonics. Computing the intersection of the curve $H K=1$ with the lower curve on the diagram $\left(K=K_{A}\right)$, we obtain $H^{2}=3 / 4$. Therefore, in the region $H^{2}>3 / 4$, the fundamental wavenumber is always unstable.

The previous discussion raises the question of the physical meaning of the upper instability region in Fig. 1. In particular, we ask whether it is possible to locate the fundamental wavenumber in the stable region (between $K_{A}$ and $K_{B}$ ), and a higher harmonic $K_{m}=m K_{0}$ (where $m$ is an integer) in the unstable upper zone 
(between $K_{B}$ and $K_{C}$ ). The constraints to be satisfied are

$$
\begin{aligned}
K_{B} & <K_{m}<K_{C}, \\
K_{0} & >K_{A} .
\end{aligned}
$$

Taking into account Eq. (45), we can write $K_{m}=m /(n H)$, and the previous inequalities become

$$
\begin{aligned}
& 2+2 \sqrt{1-H^{2}}<\frac{m^{2}}{n^{2}}<4 \\
& 2-2 \sqrt{1-H^{2}}<\frac{1}{n^{2}} .
\end{aligned}
$$

The square root can be eliminated by summing Eqs. (48)-(49). We obtain

$$
4 n^{2}-1<m^{2}<4 n^{2}
$$

which cannot be satisfied for any pair of integer numbers $(n, m)$. In summary, it is not possible to excite a harmonic in the unstable upper zone of Fig. 1, without also exciting the fundamental mode in the lower unstable region. Therefore, at least in the semiclassical regime $H<1$, we cannot expect to observe a "purely quantum" instability. However, as remarked earlier, for $H^{2}>3 / 4$ the fundamental mode is unstable, as a result of quantum effects. We stress that the above restrictions are a result of the periodic boundary conditions, and do not apply to a truly infinite plasma, for which momentum space is continuous. Still, periodic conditions can be relevant, for instance, to solid state plasmas, where the periodicity is induced by the underlying ion lattice.

\subsection{Stationary solutions - QBGK modes}

Let us now consider the stationary states of the two-stream Schrödinger-Poisson system (QBGK modes). If all quantities are dependent only on position, Eqs. (5)-(6) for $N=2$ possess the first integrals

$$
\begin{aligned}
J_{1} & =n_{1} u_{1}, \quad J_{2}=n_{2} u_{2}, \\
E_{1} & =\frac{M u_{1}^{2}}{2}-e \phi-\frac{\hbar^{2}}{2 M} \frac{d^{2}\left(\sqrt{n_{1}}\right) / d x^{2}}{\sqrt{n_{1}}}, \\
E_{2} & =\frac{M u_{2}^{2}}{2}-e \phi-\frac{\hbar^{2}}{2 M} \frac{d^{2}\left(\sqrt{n_{2}}\right) / d x^{2}}{\sqrt{n_{2}}} .
\end{aligned}
$$

We are particularly interested in the case of two symmetric streams, each carrying the same current (with opposite sign) and same kinetic energy. Therefore we write

$$
J_{1}=-J_{2}=n_{0} u_{0} / 2, \quad E_{1}=E_{2}=M u_{0}^{2} / 2
$$


where $u_{0} \neq 0$ is a nonzero reference velocity. Let $n_{1} \equiv A_{1}^{2}$ and $n_{2} \equiv A_{2}^{2}$, and transform to the dimensionless variables

$$
\begin{array}{rrr}
x^{*}=\omega_{p} x / u_{0} & , \quad A_{1,2}^{*}=A_{1,2} / \sqrt{n_{0}}, \\
\phi^{*}=\left(e \phi+E_{1}\right) / M u_{0}^{2} & , \quad H=\hbar \omega_{p} / M u_{0}^{2} .
\end{array}
$$

In these variables, the two conservation laws, Eqs. (52)-(53), and Poisson's equation (7) take the form of a six-dimensional dynamical system (we omit the stars for simplicity of notation)

$$
\begin{aligned}
H^{2} \frac{d^{2} A_{1}}{d x^{2}} & =\frac{1}{4 A_{1}^{3}}-2 \phi A_{1} \\
H^{2} \frac{d^{2} A_{2}}{d x^{2}} & =\frac{1}{4 A_{2}^{3}}-2 \phi A_{2} \\
\frac{d^{2} \phi}{d x^{2}} & =A_{1}^{2}+A_{2}^{2}-1 .
\end{aligned}
$$

Equations (57)-(59) constitute a coupled, nonlinear system of three second-order ordinary differential equations, depending on the control parameter $H$. They can be put into a Hamiltonian form, using a procedure similar to the one employed for the one-stream QBGK equations (23)-(24). This immediately provides a first integral, which is the Hamiltonian function itself (its actual expression is rather involved, and not particularly illuminating). Just as in the one-stream case, we could not find any additional constants of the motion, so that we cannot prove rigorously that the system is integrable. However, numerical integrations of Eqs. (57)-(59) (see end of this section) strongly suggest that the system is indeed integrable, with quasi-periodic solutions.

For the study of small amplitude oscillations, it suffices to expand Eqs. (57)-(59) in the vicinity of the spatially homogeneous equilibrium $A_{1}=1 / \sqrt{2}, A_{2}=1 / \sqrt{2}$, $\phi=1 / 2$. After Fourier transforming, the following system is obtained, for the perturbed quantities $A_{i}^{\prime}, \phi^{\prime}$

$$
\begin{aligned}
\left(4-H^{2} K^{2}\right) A_{i}^{\prime}+\sqrt{2} \phi^{\prime} & =0, \quad i=1,2 \\
\sqrt{2}\left(A_{1}^{\prime}+A_{2}^{\prime}\right)+K^{2} \phi^{\prime} & =0,
\end{aligned}
$$

where $K=k u_{0} / \omega_{p}$ is the dimensionless wavenumber. By searching for nontrivial solutions, one obtains the relation

$$
\left(H^{2} K^{2}-4\right)\left(H^{2} K^{4}-4 K^{2}+4\right)=0
$$

Notice that this is the same (with an equality sign) as the previously obtained Eq. (37). Solutions of Eq. (62) represent wavenumbers for which both the real and the imaginary part of the frequency vanish, and can be considered as the homogeneous 
limit of generally inhomogeneous stationary states (QBGK modes). If $H<1$ there are three such solutions, which are the wavenumbers $K_{A}, K_{B}$ and $K_{C}$ defined in Eqs. (40)-(41). If $H>1$, only the solution $K_{C}$ survives. The other two solutions become complex, so that spatially periodic QBGK modes can no longer exist.

It is also interesting to look for the eigenvectors corresponding to the eigenvalues $K_{A}, K_{B}$ and $K_{C}$. From Eqs. (60)-(61) we can express the perturbed amplitudes $A_{i}^{\prime}$ in terms of the potential. Two cases are possible : (1) if $K=K_{C}=2 / H$, then one must have $\phi^{\prime}=0$ and $A_{1}^{\prime}=-A_{2}^{\prime} ;(2)$ otherwise, if $K=K_{A}$ or $K_{B}$, we have $A_{1}^{\prime}=A_{2}^{\prime}=\sqrt{2} \phi^{\prime} /\left(H^{2} K^{2}-4\right)$. The first case is particularly interesting. It means that the mode characterized by wavenumber $K_{C}$ is a "quasi-neutral" mode, in the sense that the associated electrostatic potential is zero to first order. Indeed, one can see that no plasma parameters (such as the plasma frequency) enter the definition of $K_{C} \equiv 2 / H$. These modes can be actually accessed, for example by choosing the fundamental wavenumber $K_{0}=1 / H$, which is the largest admissible value for $K_{0}$ [see Eq. (45)]. In this case, the harmonic $2 K_{0}=2 / H$ corresponds to the quasi-neutral mode.

Numerical integration of Eqs. (57)-(59) confirms the previous results. For instance, it was verified that periodic solutions only exist for $H<1$. We take $H=0.7$ and initialize the amplitudes and the potential (at $x=0$ ) with their equilibrium value, plus a small perturbation $\epsilon$, i.e. $\phi(0)=1 / 2+\epsilon_{\phi}, A_{i}(0)=\left(1+\epsilon_{i}\right) / \sqrt{2}$. In agreement with the discussion of the previous paragraph, if we choose $\epsilon_{\phi}=0$ and $\epsilon_{1}=-\epsilon_{2}$, the wavenumber $K_{C} \simeq 2.857$ is linearly excited and thus dominates (Fig. 3), while the potential remains very small. On the other hand, if $\epsilon_{1}=\epsilon_{2}$ and $\epsilon_{\phi}$ arbitrary, modes $K_{A} \simeq 1.08$ and $K_{B} \simeq 2.645$ are linearly excited (Fig. 4). For generic perturbations, all three wavenumbers are excited. Of course these results are strictly valid only for infinitesimally small perturbations. For moderate values, other modes appear (visible on Figs. 3-4, for which $\epsilon=0.02$ ), although the linear wavenumbers are still dominant. For even larger perturbations, bounded solutions no longer exist.

\section{Time-dependent numerical simulations}

A standard numerical technique has been employed in order to integrate the timedependent Schrödinger-Poisson system. Let us write the Schrödinger equation as

$$
i \hbar \frac{\partial \psi}{\partial t}=K \psi+\Phi \psi
$$

where $K$ is the kinetic part of the Hamiltonian, and $\Phi(x, t)$ is the potential. The Hamiltonian is split into these two parts, and each is treated separately. For the 
potential part, the solution is trivial

$$
\psi^{n+1}=\psi^{n} \exp (-i \Phi \Delta t / \hbar)
$$

where $\psi^{n} \equiv \psi(n \Delta t)$, and $\Delta t$ is the timestep. For the kinetic part, we use a Crank-Nicolson scheme, which is exact to second order in $\Delta t$,

$$
i \hbar \frac{\psi^{n+1}-\psi^{n}}{\Delta t}=\frac{1}{2}(K \psi)^{n+1}+\frac{1}{2}(K \psi)^{n} .
$$

The kinetic operator $K$ is spatially discretized by using the standard centered differences formula. The time evolution is obtained by subsequently applying the potential and kinetic steps described above. Poisson's equation is solved with a Fast Fourier Transform technique just before the potential step (notice that the kinetic step does not alter the spatial density, and therefore the potential). The resulting numerical scheme is unconditionally stable and second order accurate in both space and time variables. Another crucial property of the scheme is that it conserves exactly the integral $\int|\psi|^{2} d x$ 17. A typical resolution used in the simulation is $N_{x}=512$ points, and timestep $\Delta t=0.02$.

The initial condition for the simulations is obtained by applying a sudden sinusoidal potential to the equilibrium wavefunctions given in Eq. (43). In dimensionless variables, we have

$$
\psi_{ \pm}(x, 0)=2^{-1 / 2} \exp \left( \pm i n K_{0} x\right) \exp \left(i \epsilon H^{-1} \cos \left(K_{m} x\right)\right),
$$

where $\epsilon$ and $K_{m}=m K_{0}$ are the amplitude and the wavenumber of the perturbation, and $n, m$ are integer numbers. We remind that one must have $H K_{0}=1 / n$.

Several simulations have been run in order to compare with the analytical results obtained in the linear regime, with excellent agreement between the two. As an example, we use the parameters $H=0.25$ and $n=5\left(K_{0}=0.8\right)$, and perturb the fundamental mode $(m=1)$. Figure 5 shows the evolution of two modes of the electrostatic potential. The straight line corresponds to the linear growth rate for $K_{0}$, as computed from Eq. (36), and closely matches the measured growth rate. At saturation, several modes are present.

The total momentum distribution $F(p)$ is given by the sum of the square modulus of the Fourier transform of each wavefunction, with $p=\hbar k$. As pointed out earlier, momentum space is discrete, with $\Delta p=\hbar k_{0}$ (=0.2 in the above case). For our simulations, the total momentum distribution of the unperturbed wavefunctions is simply

$$
F(p)=\frac{1}{2} \delta\left(p-M u_{0}\right)+\frac{1}{2} \delta\left(p+M u_{0}\right),
$$

where $\delta$ is the Dirac delta function. During the linear phase, the momentum distribution remains virtually unchanged, whereas at saturation we observe a significant 
spreading in momentum space, which extends to $p \simeq \pm 2.5 M u_{0}$ (Fig. 6). This is similar to the behavior of the classical two-stream instability.

As was shown in the preceding section, one cannot excite a mode in the unstable upper region of Fig. 1, without also exciting an unstable mode in the lower zone. However, if only the larger wavenumber is initially perturbed, one can hope to see it grow with the correct rate before other unstable modes can be excited. In order to do so, we take $H=0.9$ and $n=5$. The fundamental wavenumber is thus $K_{0}=2 / 9$, and is of course unstable since $K_{A}=1.18$ in this case. We only perturb the harmonic $9 K_{0}=2$, which falls within the upper unstable zone, since $K_{B}=1.883$ and $K_{A}=2.222$. Of course, several other modes are also unstable (namely, those from $K_{0}$ to $5 K_{0}$ ), but they are not initially perturbed. Figure 7 shows the evolution of mode $9 K_{0}$, which closely agrees with the result of the linear calculation for the growth rate. Several other linearly unstable modes appear at a later time, so that at saturation the spectrum is large. This is reflected in the momentum distribution, plotted on Fig. 8.

The results for the fully quantum regime, and particularly for $H \simeq 1$, are more surprising. As an example, let us take $H=1, K_{0}=1$, and perturb the fundamental mode itself. Instability occurs as expected with the correct growth rate (see Fig. 9 ). However, instead of saturating at a certain level, the system appears to decay with the same rate, and then to grow again. These nonlinear periodic oscillations do not damp even for very long times, as it has been checked numerically. Higher harmonics, which are linearly stable, are driven by the fundamental mode, and show a similar pattern, although at a lower level. The period of the oscillations is not universal, and depends on the amplitude of the initial perturbation, which confirms that this is indeed a nonlinear effect. A similar, although less pronounced behavior, is also observed for $H>1$. The momentum distribution is virtually unchanged over the entire duration of the simulation. It must be noted that, for parameters such that $H K_{0}=1$, the minimum nonzero value of the momentum is $\left|p_{\text {min }}\right|=M u_{0}$, and is therefore equal to the momentum of the unperturbed streams. In other words, the streams occupy the lowest possible level in momentum space. Although we do not have a detailed explanation for this phenomenon, it appears to be an example of a completely reversible quantum system, in which the initial condition is almost perfectly reconstructed after one period [18]. This is to be compared with the inherently irreversible classical dynamics, for which returning to the initial state after saturation is virtually impossible.

Finally, we have studied the evolution of perturbed QBGK equilibria. It is particularly instructive to consider the case where only the $K_{C}=2 / H$ mode is present. This, as detailed in the previous section, is a "quasi-neutral" mode, in the sense that the associated electrostatic potential is zero to first order in the perturbation parameter. We construct a weakly inhomogeneous equilibrium by using the form 
of Eq. (3) for the wavefunctions $\psi_{1,2}$. Both the amplitude and the phase should be sinusoidal functions. For the amplitudes, we have

$$
A_{1,2}=\frac{1}{\sqrt{2}}\left(1 \pm \epsilon \cos \left(K_{C} x\right)\right)
$$

where $K_{C}=m K_{0}$ is the wavenumber of the QBGK mode; $m$ therefore represents the number of density oscillations. The phases $S_{1,2}$ are obtained from the definition of Eq. (4), and remembering that, in dimensionless units, $u_{i} n_{i}= \pm 1 / 2$. One obtains, to first order in $\epsilon$

$$
S_{1,2}= \pm x-2 \epsilon K_{C}^{-1} \sin \left(K_{C} x\right)
$$

By virtue of the relations $n H K_{0}=1$ and $K_{C}=m K_{0}=2 / H$, we obtain that $m=2 n$ must be an even number.

As an example, we take $H=0.5, K_{C}=4$. With $n=1$, we obtain $K_{0}=2$, and the number of spatial oscillations is $m=2 n=2$. Notice that, in this case, the fundamental wavenumber is stable. If we had chosen $n=2$, we would have had $K_{0}=1$, which is unstable. With this value of $H$, it is therefore possible to construct a stable QBGK mode displaying at most two spatial oscillations. In order to have more oscillations, a smaller value of $H$ should be used. For small values of the perturbation parameter $\epsilon$, the simulations confirm the linear analytical results, and virtually no evolution is observed; besides, the potential fluctuations stay small compared to the density ones. For larger values of the perturbation $(\epsilon=0.1)$, some temporal variations are observed (Fig. 10), since the wavefunctions given by Eqs. (68)-(69) no longer represent an exact stationary state. However, the periodic structure is not destroyed, and the potential fluctuations remain an order of magnitude smaller than the density fluctuations. These results show that a quantum plasma can support almost stationary, quasi-neutral, periodic solutions. These display significant density fluctuations for each stream, but small potential fluctuations. They have no analog in a classical two-stream plasma.

\section{Conclusion}

In this work, we have introduced a quantum multistream model to describe some physical phenomena arising in quantum plasmas. The quantum multistream model may be considered as a discrete version of the Wigner-Poisson model, in the same sense as the classical multistream model is a discrete form of the Vlasov-Poisson system. Indeed, it is well known [19 that the Wigner-Poisson system is formally

equivalent to an infinite set of Schrödinger equations, coupled by a scalar potential obeying Poisson's equations. However, it is often more appropriate to work with 
the hydrodynamic formulation of quantum mechanics, since it makes direct use of the same physical quantities that are employed in classical physics (density, velocity, pressure). Moreover, the stability analysis and perturbation calculations become straightforward in the hydrodynamic formulation. On the other hand, we have used the Schrödinger representation for the time-dependent simulations, since accurate numerical techniques for this equation are well-known from the computational literature.

For the case of the two-stream instability, it has been shown that the dispersion relation possesses three branches, one of a semiclassical character and two of a purely quantum nature. It is interesting to observe that even the classical branch reveals some unexpected features: for small $H$, quantum effects tend to enhance the two-stream instability. More precisely, some classically stable wavenumbers are destabilized for sufficiently large values of $H<1$. On the other hand, strong quantum effect can yield the opposite result: for $H>2$, some classically unstable wavenumbers become stable. The purely quantum region of the dispersion relation (the upper unstable zone of Fig. 1) cannot however be excited without also exciting some wavenumber in the lower (semiclassical) region. This means that a purely quantum instability cannot be observed for $H<1$. Extensive numerical simulations, run for different values of the relevant parameters, wholly support the analytical results obtained from linear theory. In the fully quantum case, we have observed a surprising, yet unexplained, regime of undamped nonlinear oscillations. This is a purely quantum effect, which is probably linked to quantum recurrences and echoes [18.

We have also considered the stationary states of the Schrödinger-Poisson system, which can be viewed as the quantum analog of the classical BGK modes. Such QBGK modes are described by a nonlinear system of coupled second-order differential equations, parametrized by the dimensionless Planck's constant $H$. Such system provides an adequate framework for the analysis of QBGK modes, a task which would be rather difficult in the Wigner-Poisson formalism. In particular, numerical simulations have shown that quasi-neutral, spatially periodic, stationary states can be created in the two-stream plasma, and can survive over long times.

Some interesting questions remain to be addressed. In the present work, we have considered in detail only the one and two-stream cases. Further investigations are needed to explain the properties of the quantum multistream model when the number of streams is large. In this case, the Wigner-Poisson system could be a more appropriate model, despite its intrinsic mathematical difficulties. However, an intermediate number of streams might be sufficient to describe the main physical phenomena. Linear and nonlinear Landau damping, for example, should be good candidates to test these ideas, both analytically and numerically. 


\section{Acknowledgments}

We would like to thank Pierre Bertrand for his valuable comments and suggestions. One of us (F. H.) thanks the Laboratoire de Physique des Milieux Ionisés for the hospitality while this work was carried out, and the Brazilian agency Conselho Nacional de Desenvolvimento Cientifico e Tecnólogico (CNPq) for financial support. 


\section{References}

[1] A. Arnold and H. Steinrück, J. Appl. Math. and Phys. 40, 793 (1989).

[2] B. Shokri and A. A. Rukhadze, Phys. Plasmas 6, 3450 (1999); B. Shokri and A. A. Rukhadze, Phys. Plasmas 6, 4467 (1999).

[3] N. C. Kluksdahl, A. M. Kriman, D. K. Ferry and C. Ringhofer, Phys. Rev. B 39, 7720 (1989).

[4] M. C. Yalabik, G. Neofotistos, K. Diff, H. Guo and J. D. Gunton, IEEE Trans. Electron Devices 36, 1009 (1989).

[5] I. H. Tan, G. L. Snider, L. D. Chang and E. L. Hu, J. Appl. Phys. 68, 4071 (1990).

[6] S. Mola, G. Manfredi and M. R. Feix, J. Plasma Phys. 50, 145 (1993).

[7] J. H. Luscombe, A. M. Bouchard and M. Luban, Phys. Rev. B 46, 10262 (1992).

[8] E. P. Wigner, Phys. Rev. 40, 749 (1932).

[9] J. E. Moyal, Proc. Cambridge Phil. Soc. 45, 99 (1949).

[10] V. I. Tatarski, Sov. Phys. Usp. 26, 311 (1983).

[11] J. Dawson, Phys. Fluids 4, 869 (1961).

[12] I. B. Bernstein, J. M. Greene and M. D. Kruskal, Phys. Rev. 108, 546 (1957).

[13] J. E. Drummond, Plasma Physics (McGraw- Hill, New York, 1961).

[14] P. Bertrand, V. T. Nguyen, M. Gros, B. Izrar, M. R. Feix and J. Gutierrez, J. Plasma Phys. 23, 401 (1980).

[15] V. I. Arnold, Mathematical Methods of Classical Mechanics (Springer, New York, 1978).

[16] N. Suh, M. R. Feix and P. Bertrand, J. Comput. Phys. 94, 403 (1991).

[17] G. Manfredi and M. Ottaviani, in Dynamical Systems, Plasmas and Gravitation, edited by P.G.L. Leach, S.E. Bouquet, J.-L. Rouet, E. Fijalkow (Springer, Berlin, 1999), pp. 82-92.

[18] G. Manfredi and M. R. Feix, Phys. Rev. E 53, 6460 (1996).

[19] P. A. Markowich, Math. Meth. Appl. Sci. 11, 459 (1989). 


\section{Figure Captions}

Figure 1 : Stability diagram for the two-stream plasma. The filled area is unstable. The dashed line corresponds to $H K=1$. Lower and middle solid curves: $K_{A}^{2}$ and $K_{B}^{2}$ as defined in Eq. (40). Upper solid curve: $K_{C}^{2}$ as defined in Eq. (41).

Figure 2: Plot of the squared growth rate $\gamma$ as a function of the dimensionless wavenumber $K$, for different values of Planck's constant. $H=0.5$, solid line; $H=1$, dashed line; $H=2$, dotted line. The intersections of these curves with the $K$ axis correspond to wavenumbers $K_{A}, K_{B}$ and $K_{C}$ as defined in Eqs. (40)-(41). For $H=0.5$, only the intersection at $K_{A} \simeq 1.035$ is shown; the intersections at $K_{B} \simeq 3.864$ and $K_{C}=4$ are outside the $K$ axis range. For $H=1$, the intersections are at $K_{A}=K_{B}=\sqrt{2}$ and $K_{C}=2$. For $H=2$, there is only one intersection at $K_{C}=1$.

Figure 3 : Stationary solution of the two-stream Schrödinger-Poisson plasma (QBGK mode), with $H=0.7, \epsilon_{\phi}=0, \epsilon_{1}=-\epsilon_{2}=0.02$. (a) Spatial variation of the density fluctuations $A_{1}^{\prime}$ (solid line), $A_{2}^{\prime}$ (dashed line), and potential fluctuations $\phi^{\prime}$ (dotted line). Notice that the potential remains small. (b) Fourier transform of $A_{1}^{\prime}$ : the linear wavenumber $K_{C} \simeq 2.857$ is dominant.

Figure 4 : Stationary solution of the two-stream Schrödinger-Poisson plasma (QBGK mode), with $H=0.7, \epsilon_{\phi}=0, \epsilon_{1}=\epsilon_{2}=0.02$. (a) Spatial variation of the density fluctuations $A_{1}^{\prime}$ (solid line), $A_{2}^{\prime}$ (dashed line), and potential fluctuations $\phi^{\prime}$ (dotted line). Note that the solid and dashed lines are superposed, since $A_{1}^{\prime} \simeq A_{2}^{\prime}$. (b) Fourier transform of $A_{1}^{\prime}$ : the linear wavenumbers $K_{A} \simeq 1.080$ and $K_{B} \simeq 2.645$ are dominant.

Figure 5: Two-stream instability - evolution of the fundamental mode $K_{0}=0.8$ (solid line), and first harmonic $2 K_{0}$ (dashed line) of the electrostatic potential, for $H=0.25$. The straight line corresponds to the growth rate of $K_{0}$ computed from linear theory, $\gamma=0.3116$.

Figure 6: Momentum distribution for the same case of Fig. 5, at times $t=0$ (dotted line) and $t=80$ (solid line). The final distribution has been magnified by a factor two. Momentum space is discrete with $\Delta p=\hbar k_{0}=0.2$.

Figure 7 : Two-stream instability - evolution of mode $9 K_{0}$ of the electrostatic potential, for $H=0.9$ and $K_{0}=2 / 9$. The straight line corresponds to the growth rate computed from linear theory, $\gamma=0.1085$.

Figure 8: Momentum distribution for the same case of Fig. 7, at times $t=0$ (dotted line) and $t=160$ (solid line). The final distribution has been magnified by a factor two. Momentum space is discrete with $\Delta p=\hbar k_{0}=0.2$. 
Figure 9: Two-stream instability - evolution of the fundamental mode $K_{0}=1$ (solid line), and first harmonic $2 K_{0}$ (dashed line) of the electrostatic potential, for $H=1$. The straight line corresponds to the growth rate of mode $K_{0}$ computed from linear theory, $\gamma=0.2297$.

Figure 10 : Evolution of a strongly nonlinear $(\epsilon=0.1)$ quasi-neutral $\left(K=K_{C}=\right.$ 4) QBGK equilibrium, for the set of parameters: $H=0.5, K_{0}=2$. The number of spatial oscillations is $m=2$. The density fluctuations $n_{1}^{\prime}$ (solid line) and $n_{2}^{\prime}$ (dashed line), and potential fluctuations $\phi^{\prime}$ (magnified by a factor 100, dotted line), are shown at different times. 


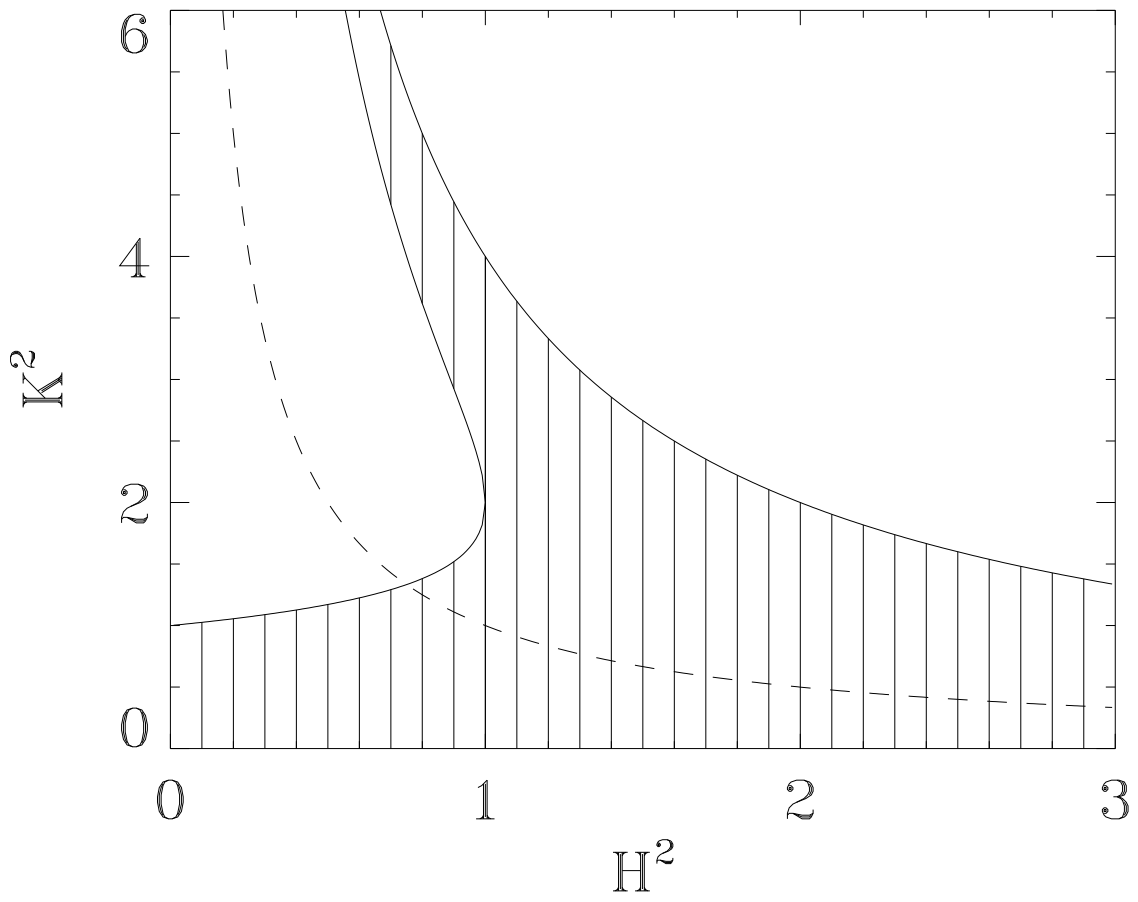

Figure $\mathbb{1}$, Phys. Rev. E, Haas 


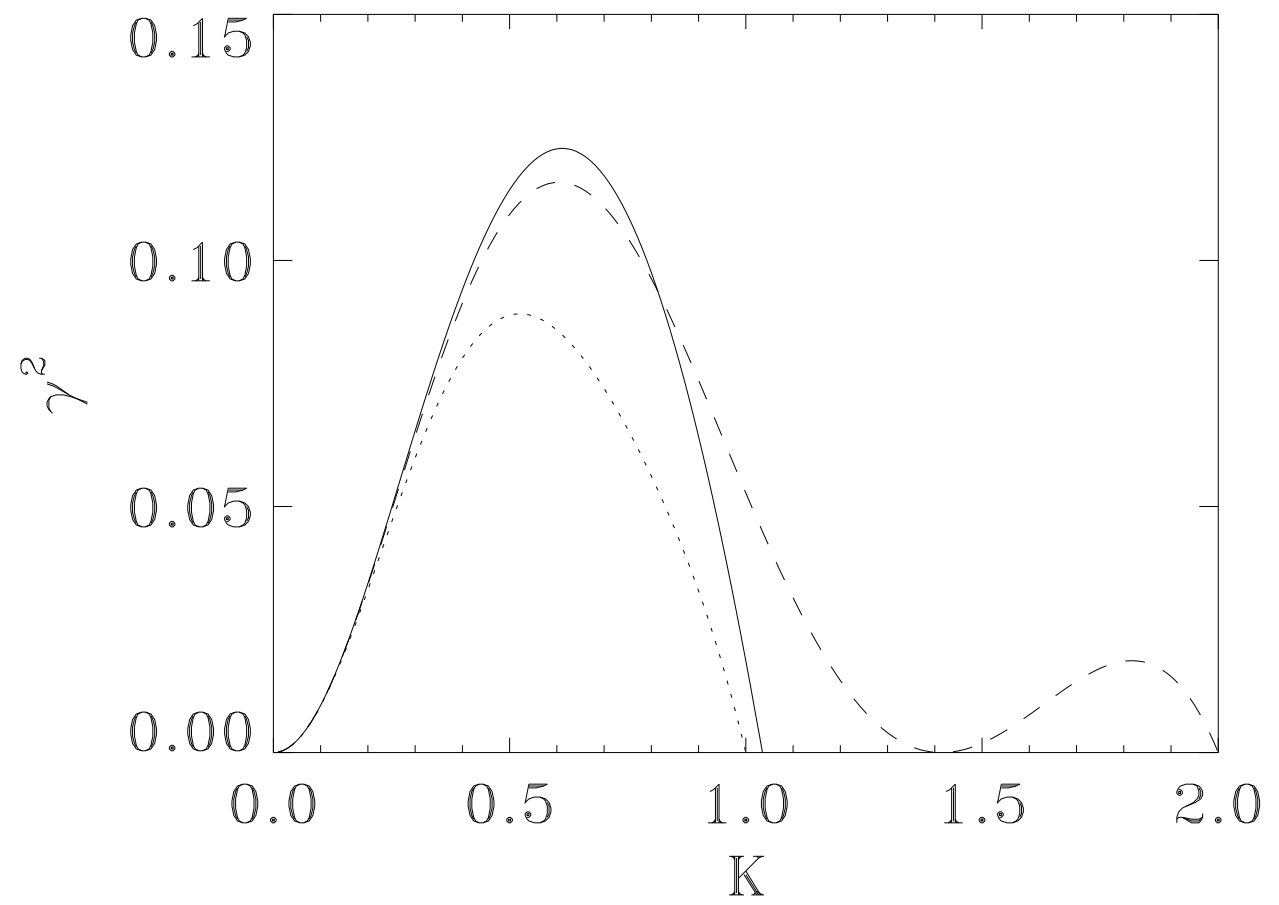

Figure 2, Phys. Rev. E, Haas 

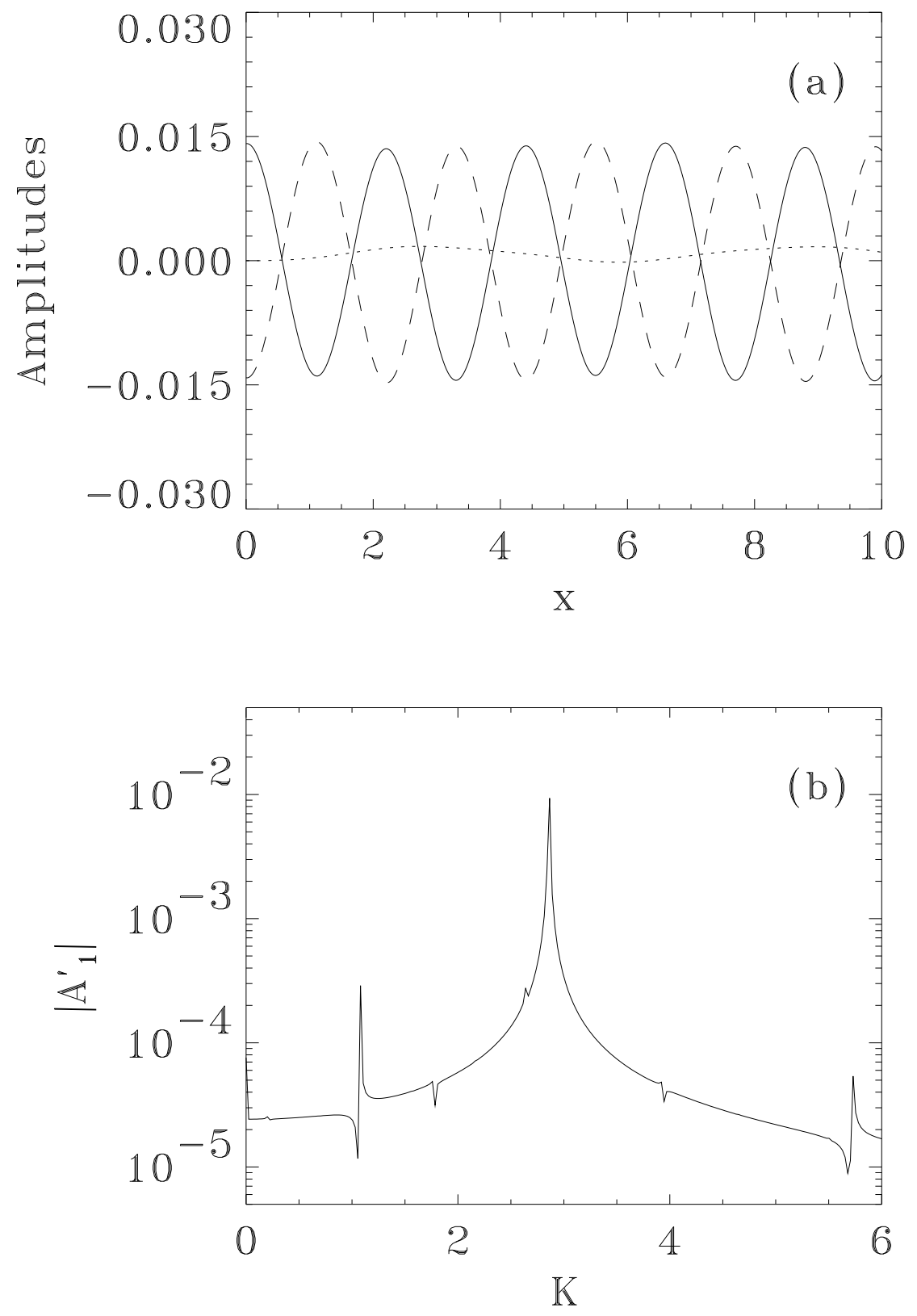

Figure 3, Phys. Rev. E, Haas 

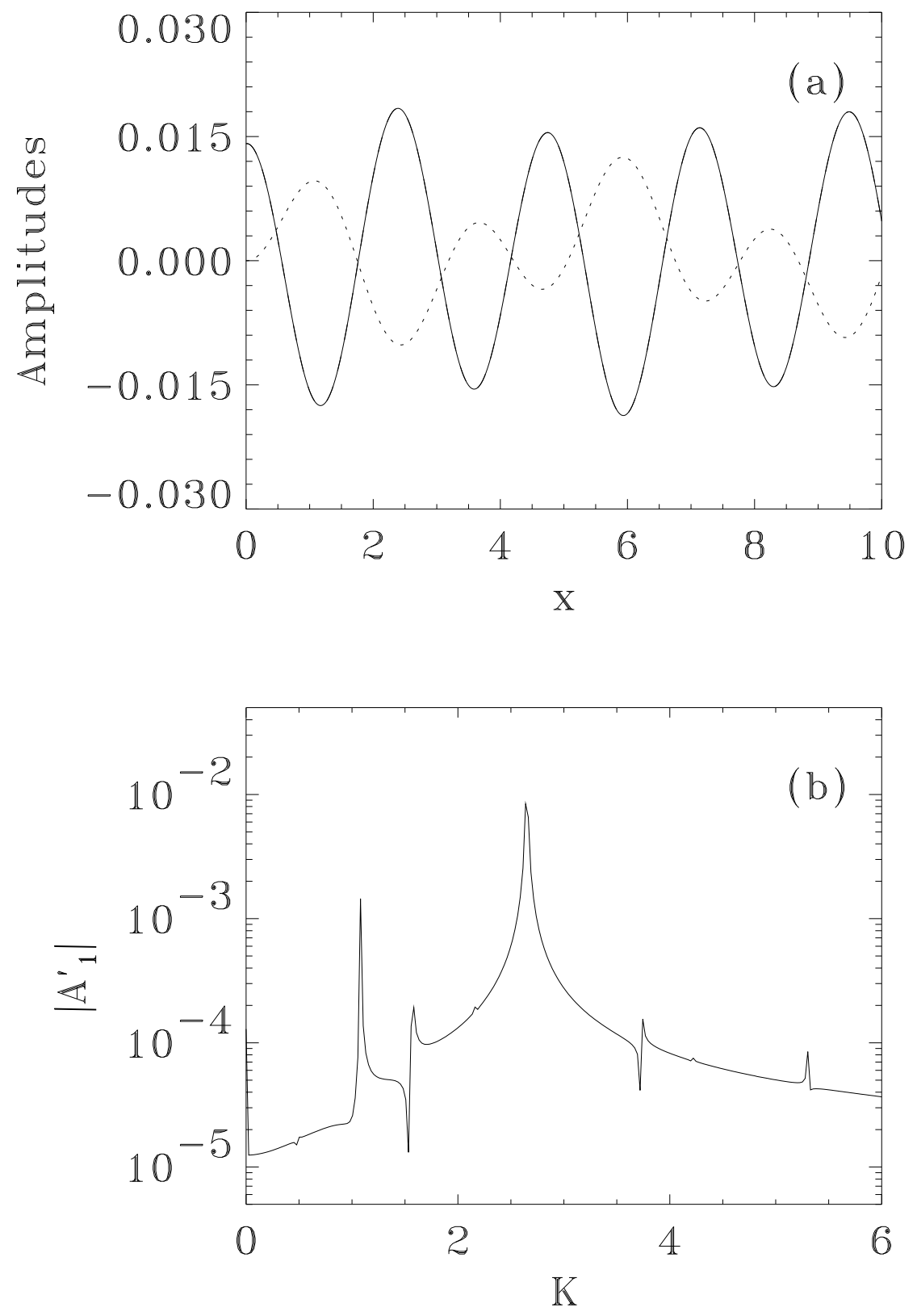

Figure 4, Phys. Rev. E, Haas 


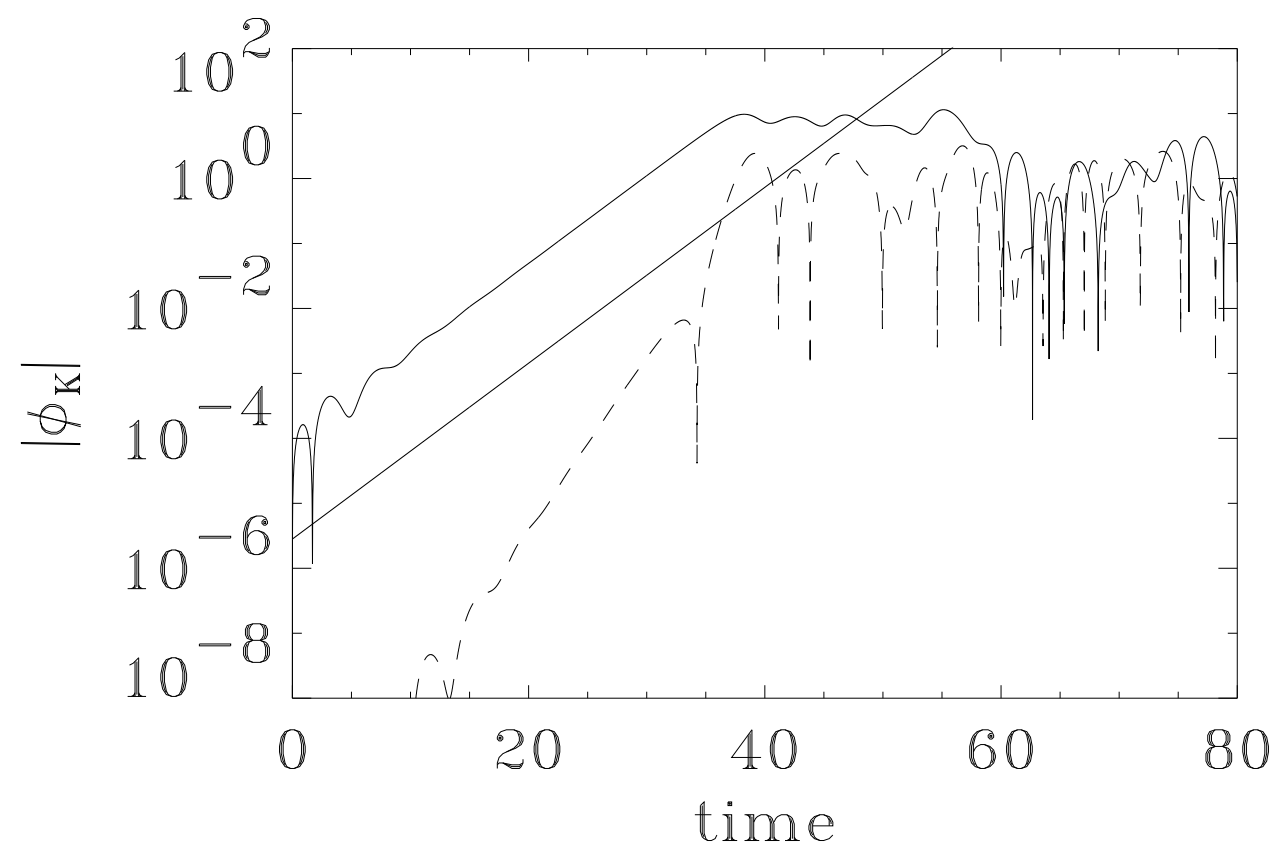

Figure 5, Phys. Rev. E, Haas 


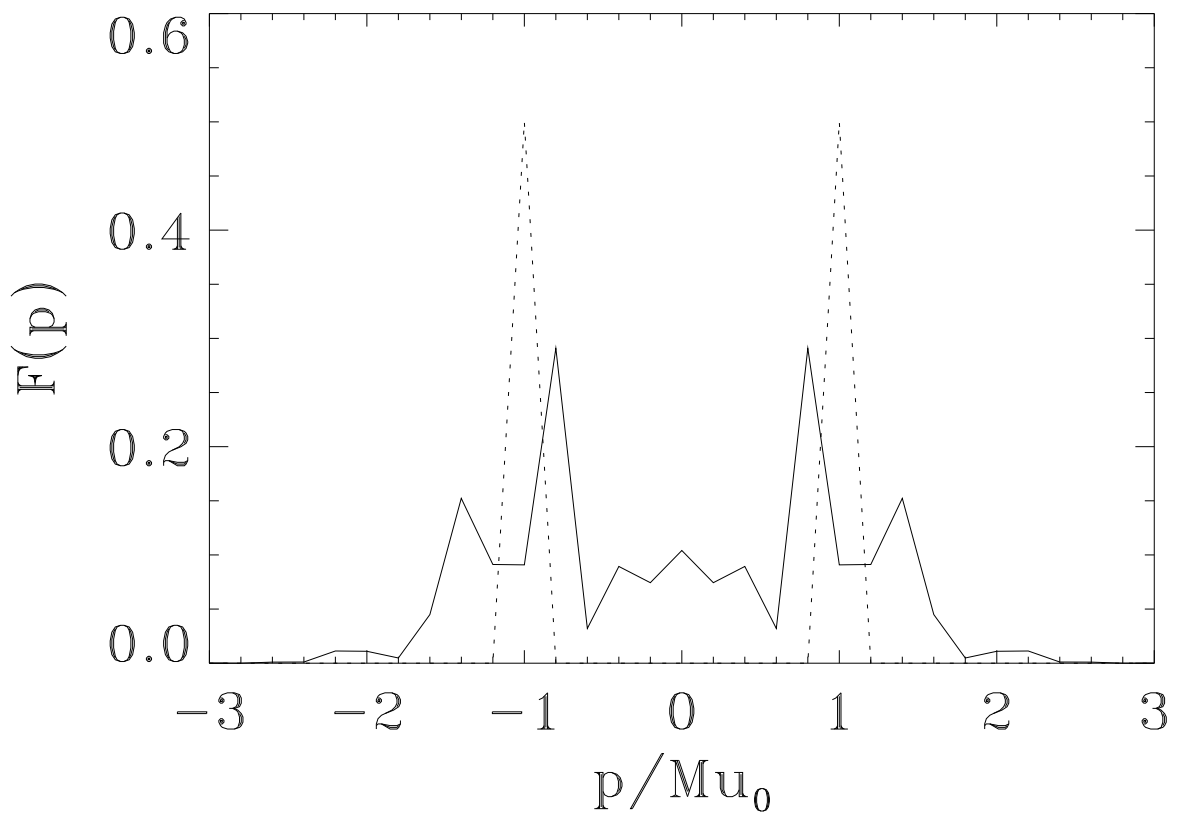

Figure 6, Phys. Rev. E, Haas 


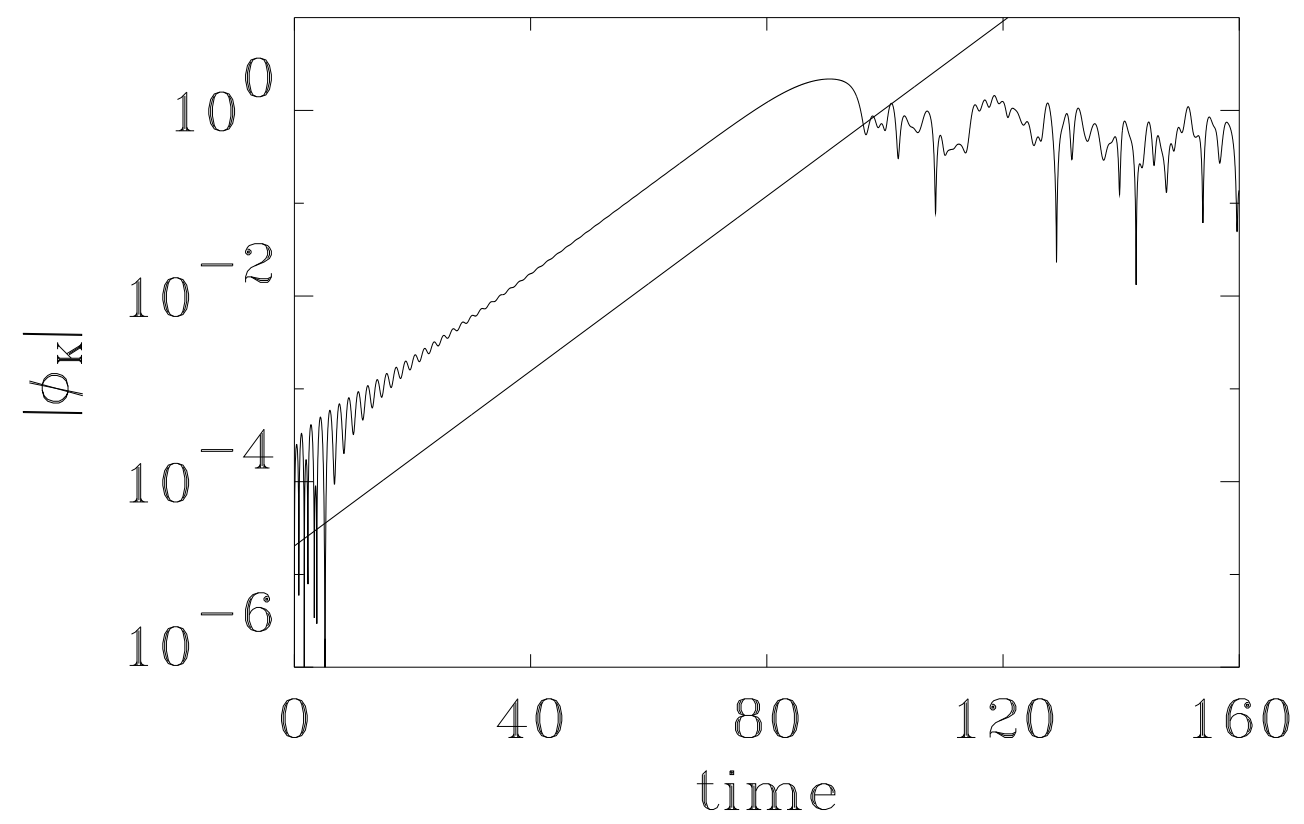

Figure 7, Phys. Rev. E, Haas 


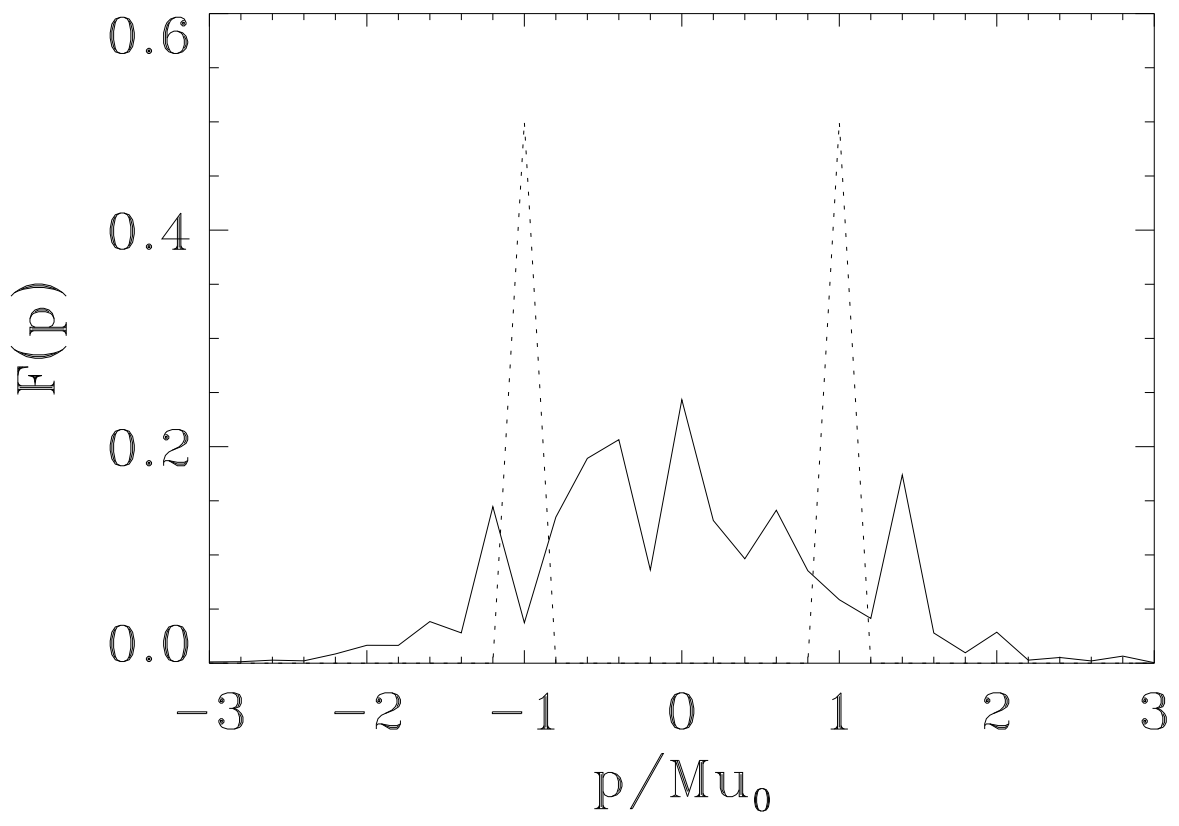

Figure 8, Phys. Rev. E, Haas 


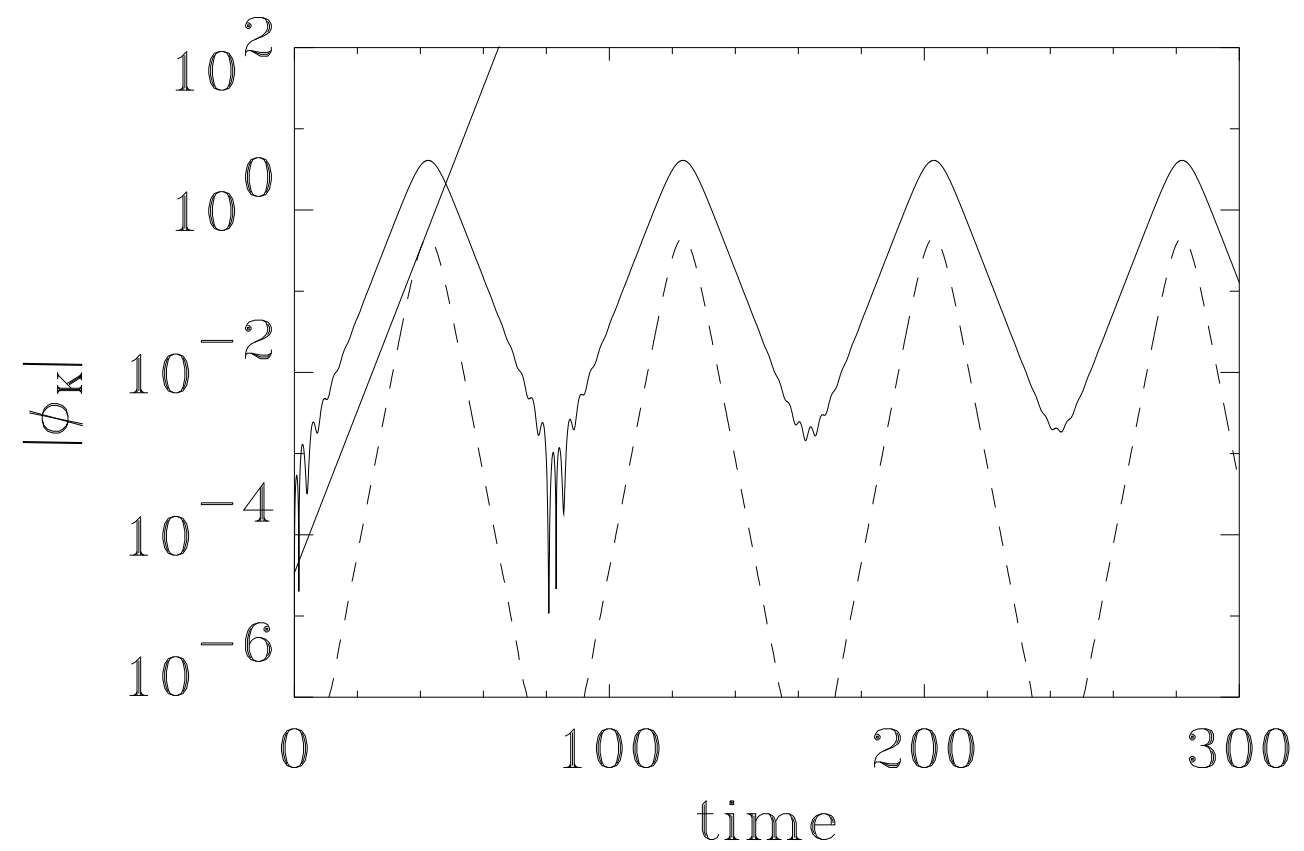

Figure 9, Phys. Rev. E, Haas 

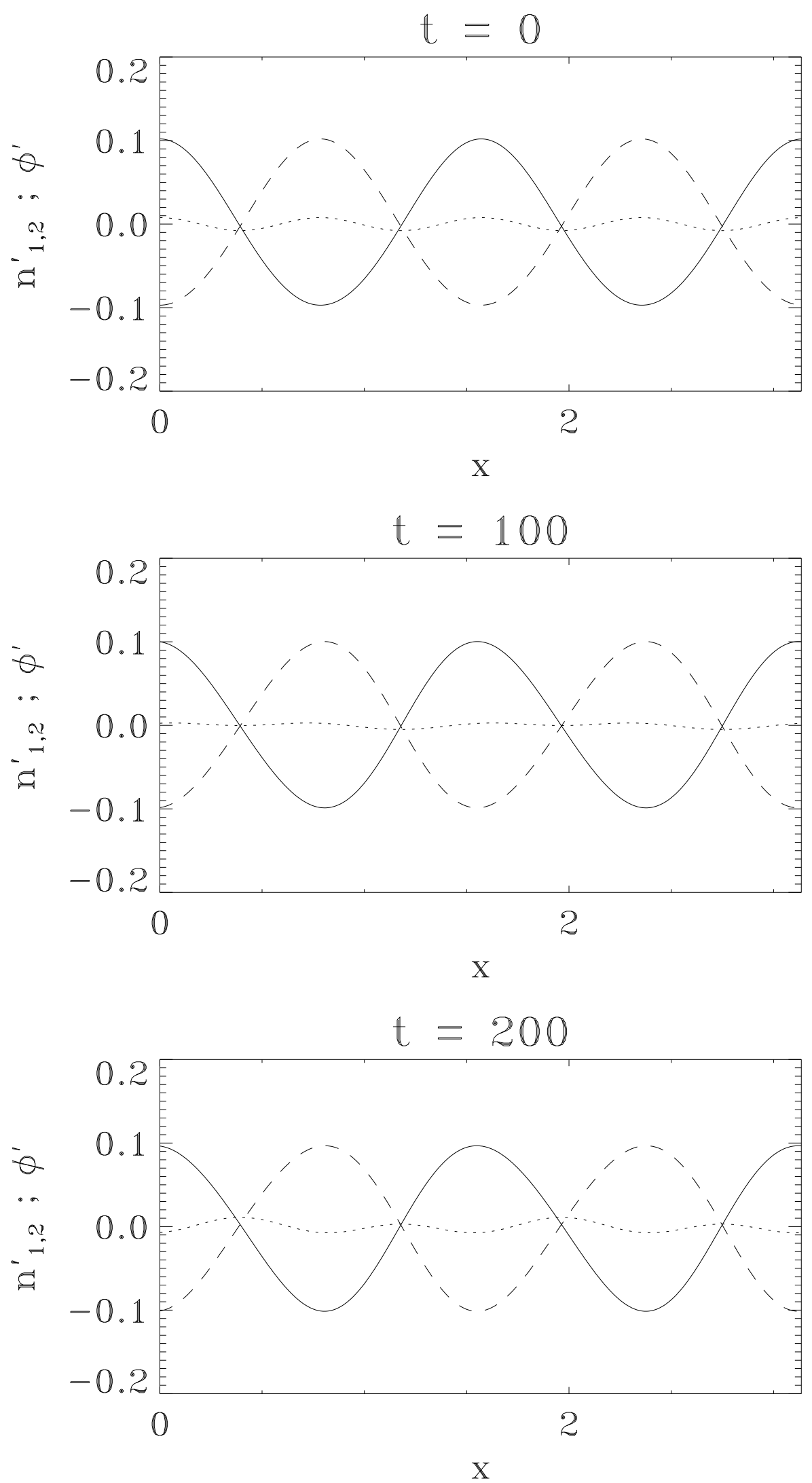

Figure 10, Phys. Rev. E, Haas 